\title{
Graphitization of Diamond by Means of UV Laser Writing: A Transmission Electron Microscopy Study
}

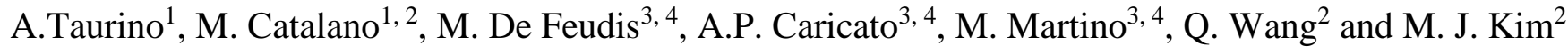 \\ 1. CNR IMM, Institute for Microelectronics and Microsystems, Via Monteroni, Lecce, Italy. \\ 2. Dep. of Materials Science and Engineering, Univ. of Texas at Dallas, Richardson, Texas, USA. \\ 3. Department of Mathematics and Physics, University of Salento, Lecce, Italy. \\ 4. INFN National Institute of Nuclear Physics, Lecce, Italy.
}

Diamond and related devices represent a field of great scientific and technological interest, due to both the interesting physical properties of diamond, such as the radiation hardness, high thermal conductivity, large bandgap, high carrier mobility, chemical stability, bio-inertness and biological tissue equivalence, and the application of diamond devices in sensors, nuclear physics and medicine[1,2]. In order to exploit the great potentials of diamond sensors, having good electrical contacts is essential. Contact adhesion, stability, radiation hardness and ohmic behaviour are generally crucial issues of surface metallization for contact realization. Laser-induced graphitization is an alternative single step process that could be used to produce bidimensional conductive structures fully integrated in all-carbon devices [3].

In this work, strip- and spot-like graphitic structures were produced on high quality thermal grade CVD diamond surface (10x10x0.25 $\left.\mathrm{mm}^{3}\right)$ by using an ArF excimer UV laser beam (wavelength $193 \mathrm{~nm}$, pulse duration $20 \mathrm{~ns}$ and a repetition rate $10 \mathrm{~Hz}$ ). Different values of laser energy density and number of laser cycles were used to study the evolution from diamond to graphite at different irradiation conditions. The sample was irradiated in air and at room temperature. Spots with increasing number of laser pulses $(\mathrm{N}=1,8,100)$ and laser fluence $\left(\mathrm{F}=5\right.$ and $\left.7 \mathrm{~J} / \mathrm{cm}^{2}\right)$, and strips with several up-and down laser scan cycles $(\mathrm{C}=2,12)$ and same laser fluence as spots, were obtained. A Scanning Electron Microscopy (SEM) study was performed on the graphitic structures in order to correlate their morphology to the laser process. For a better understanding of the diamond-graphite transition. The strip obtained at the lowest irradiation condition was selected for analysis by Transmission Electron Microscopy (TEM) and Scanning Transmission Electron Microscopy (STEM). A Zeiss NVISION 40 dual beam SEM-FIB instrument was used for SEM investigations whereas the TEM/STEM analyses were performed by using a JEMARM200F TEM/STEM instrument, equipped with a spherical aberration (Cs) corrector (CEOS GmbH, Heidelberg, Germany) and operated at $200 \mathrm{kV}$. TEM cross-sectional samples were prepared with a FEI Nova 200 dual-beam FIB/SEM by using a modified lift-out method. The preparation of diamond based lamellas is not straightforward, as the sample heavily charges during the electron beam imaging and during the milling procedure, making it difficult to exactly locate the area of interest during the entire lamella preparation procedure. The diamond etching rate is very low, therefore relatively high currents need to be used, often resulting in damage to the lamella. In order to reduce the charging effect, the Omniprobe tip was used and put in contact with the sample, very close to the milling/etching regions, till the lamella lift out. This allowed to stabilize the image and to obtain the lamella, but resulted in much longer processing times.

Figure 1 shows the SEM images of strip ( $a, b)$ and spot structures (c, d) obtained at different irradiation conditions. In particular, the strip was obtained at the lowest irradiation conditions, whereas the spots were realized at a laser fluence of $7 \mathrm{~J} / \mathrm{cm}^{2}$ and with a number of pulses of 8 and 100. The images in Figure $1 \mathrm{a}, \mathrm{c}$, and $\mathrm{d}$ were obtained in tilt view (tilt angle $50^{\circ}$ ) and evidence the ablation effect induced by the laser beam, which strongly increases with the number of cycles (spots). The full strip image is shown 
as inset of figure $1 \mathrm{a}$, whereas the fine morphology of the strip is shown in figure $1 \mathrm{~b}$; it is worth noting that the laser ablation decorates the surface with structures resulting from the different ablation rate of grains with different orientation. In Figure 2 a, an overview of the cross-section is presented, highlighting the different layers of the sample and of the lamella preparation. In figure $2 \mathrm{~b}$, the graphitized layer is clearly visible, having a variable thickness between $150 \mathrm{~nm}$ and $500 \mathrm{~nm}$. The surface of the graphitized layer as well as its interface with diamond is highly corrugated as a results of the competition between ablation and damage propagation, probably depending on the diamond grain orientation. The high resolution image in figure $2 \mathrm{c}$ shows the diamond-graphite interface, where the $\{002\}$ graphite and $\{111\}$ diamond lattice planes are observed. The misorientation of the $\{002\}$ planes of the graphite layer can be inferred from the FFT pattern, shown in the inset.

These results demonstrate the efficacy of the laser process to obtain good quality diamond integrated conductive layers of graphite to be used as contacts for the realization of diamond sensors; nevertheless the thickness variation, resulting from the competition between ablation and damage propagation, and the local variation of the graphite $\{002\}$ planes orientation, inferred from the TEM results, are expected to influence the electrical conductivity of the contacts; suitable laser writing parameters have to be selected in order to optimize the transport properties of the graphitic layers.

\section{References:}

[1] E Alemanno et al. Diam. Relat. Mater. 38 (2013) p. 32

[2] M Bruzzi, et al. Diam. Relat. Mater. 20 (2) (2011) 84.

[3] M. De Feudis et al. Diam. Relat. Mater. 75 (2017) 25.
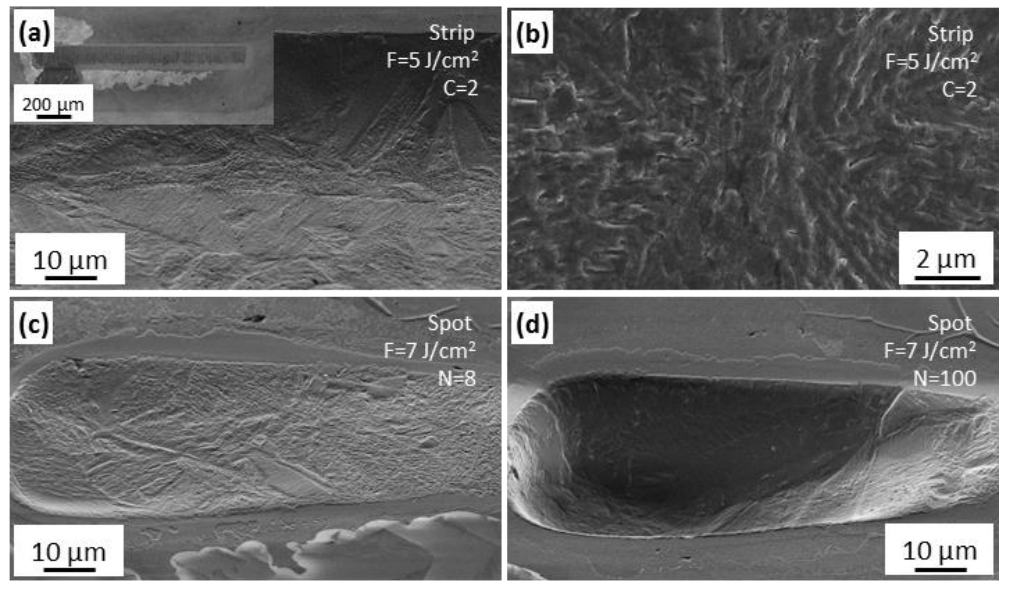

Figure 1: SEM images of the two types of diamond graphitized structures, i.e. $(a, b)$ strip obtained at $\mathrm{F}=5 \mathrm{~J} / \mathrm{cm}^{2}$, and $\left.\mathrm{C}=2\right)$ and $(\mathrm{c}, \mathrm{d})$ two spots obtained at $\mathrm{F}=7 \mathrm{~J} / \mathrm{cm}^{2}$, and $\mathrm{N}=8$ and $\mathrm{N}=100$.
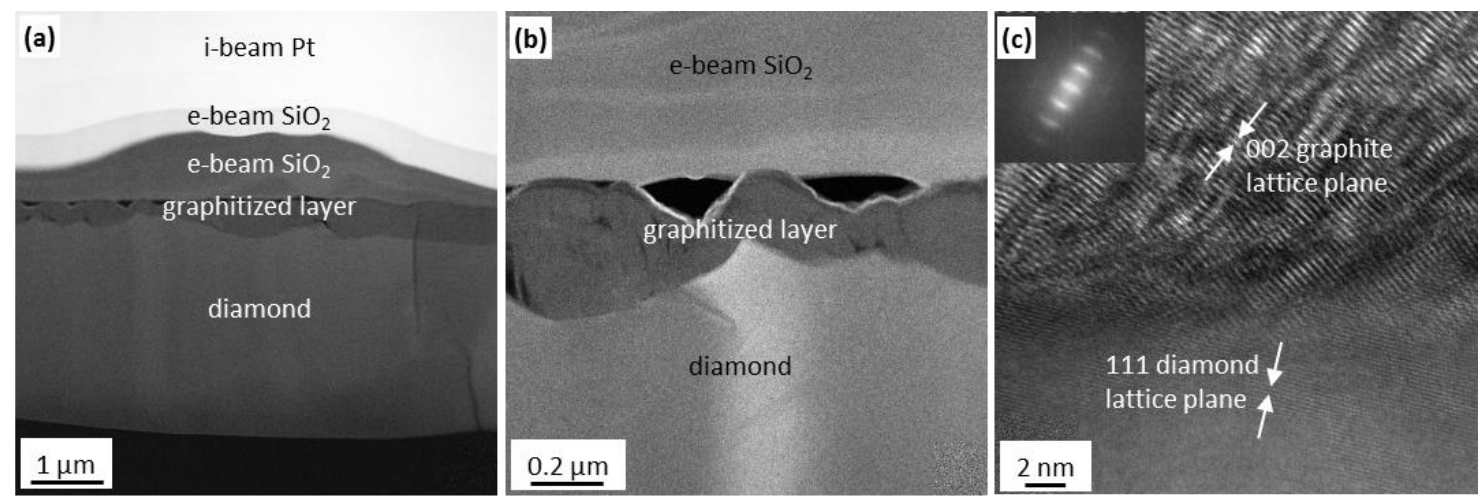

Figure 2: STEM images $(a, b)$ of a lamella prepared from the strip at the lowest irradiation conditions and high resolution TEM image of the graphite-diamond interface. 